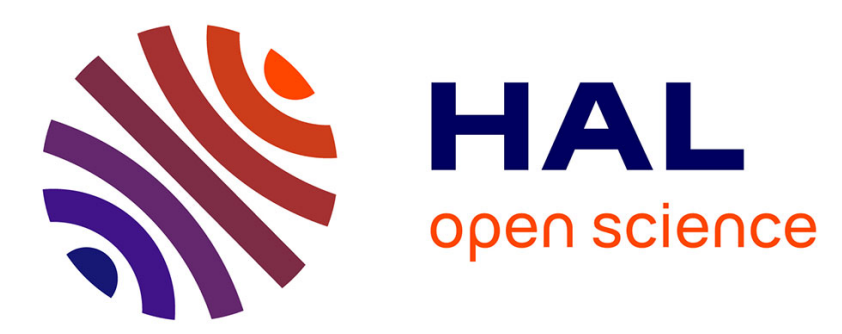

\title{
La resensibilisation, aux basses températures, des photopiles au sélénium par l'action du proche infra-rouge \\ G. Blet
}

\section{To cite this version:}

G. Blet. La resensibilisation, aux basses températures, des photopiles au sélénium par l'action du proche infra-rouge. J. Phys. Radium, 1957, 18 (2), pp.121-127. 10.1051/jphysrad:01957001802012100 . jpa-00235625

\section{HAL Id: jpa-00235625 https://hal.science/jpa-00235625}

Submitted on 1 Jan 1957

HAL is a multi-disciplinary open access archive for the deposit and dissemination of scientific research documents, whether they are published or not. The documents may come from teaching and research institutions in France or abroad, or from public or private research centers.
L'archive ouverte pluridisciplinaire HAL, est destinée au dépôt et à la diffusion de documents scientifiques de niveau recherche, publiés ou non, émanant des établissements d'enseignement et de recherche français ou étrangers, des laboratoires publics ou privés. 


\title{
LA RESENSIBILISATION, AUX BASSES TEMPERATURES, DES PHOTOPILES AU SÉLÉNIUM PAR L'ACTION DU PROCHE INFRA-ROUGE.
}

\author{
Par (i. BLET.

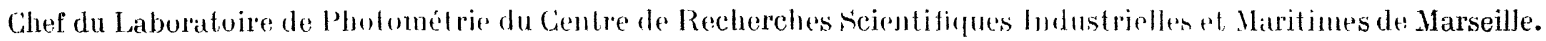

\section{Description du phénomène. - Notations.}

Au cours des mesures effectués pour déterniner la ('ourbe de sensibilité spectrale de photopiles au sélénium aux basses températures, nous avons constaté que les résultats ohtenus dépendaient dans une tres large mesure des conditions opératoires. Nous avons pu mettre au point une technique de mesure donnant à coup sûr des résultats reproductibles que nous avons publiés par ailleurs.

Le phénomène nouveau que nous avons mis en évidence est le suivant : "Lorsqu'on refroidit à partir de la température ambiante une photopile au sélénium, sa sensibilité, pour une longueur d'onde donnée, décroit très fortement. L'envoi sur ladite cellule d'un flux d'énergie dans le domaine dı proche infrarouge fait remonter progressivement la sensibilité jusqu'à une valeur assez peu diflérente de la valeur initiale. ")

Ce phénomène est évidemment assez complexe vu le nombre de paramètres qui interviennent. Nous allons tout d'abord préciser les notations utilisées au cours de cette étude.

$\lambda=$ Longueur d'onde pour laquelle on mesure la sensibilité.

$\sigma_{0 \lambda}=$ Sensibilité pour la longueur d'onde $\lambda$, à la température de $273^{\circ} \mathrm{K}\left(0^{\circ} \mathrm{C}\right)$.

$\sigma_{\tau_{\lambda}}=$ Sensibilité pour la longueur d'onde $\lambda, \dot{a}$ la température de refroidissement $T$.

$\sigma_{\lambda}=$ Sensibilité pour la longueur d'onde $\lambda$, en cours de resensibilisation.

$\sigma_{\infty \lambda}=$ Sensibilité pour la longueur d'onde $\lambda$, valeur limite obtenue par resensibilisation.

$T=$ Température de refroidissement.

$\lambda_{k}=$ Longueur d'onde utilisée pour la resensibilisation.

$\theta_{k}=$ Durée d'exposition au rayonnement de resensibilisation.

$W_{l i \lambda}=$ Intensité en watts du flux resensibilisateur de longueur d'onde $\lambda_{l}$.

$E_{R i}=$ Énergie totale en ergs ou en électronvolts, nécessaire pour resensibilisation totale par un ravonnement de longueur d'onde $\lambda_{l i}$.

l. B. - Lindice $\lambda$ ne sera adjoint aux diverses notalions ri-dessus qu'on cas de nécessité.
11. Influence des divers paramètres. -.. ('e phéno)urène de resensibilisation ou réactivation fait intervenir un assez grand nombre de paramètres-dont nous étudierons à tour de role J"influence. Mentiomons tout d'abord la longueur d'onde $\lambda$, pour laquelle on mesure la sensililité ainsi que la température $T$ à laquelle la cellule est ensuite refroidie. Pour la réactiver nous utiliserons wo rayonnement dont nous pouvons faire varier la longueur d'onde $\lambda_{R}$, l'intensité $W^{\prime}$, et la durée d'application. Signalons tout de suite que le phénomène étudié comporte trois phases : premièrement une phase de croissance, deuxiemement une phase stable, troisièmement une phase de décroissance. la phase stable est caractérisée par la sensibilité limite obtenue par réactivation.

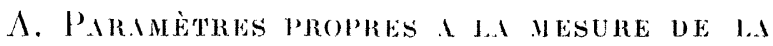
SENSIBILITÉ : $\lambda$ et $T .-\Lambda$ la température ordinaire, la détermination de la courbe de sensibilité spectrale ne comporte pàs de difficultés particulières, mais dès que l'on opère à une température inférieure il convient de prendre certaines précautions. En effet, pour éviter les phénomènes de resensibilisation qui forment précisément le sujet de cette étude, il faut effectuer les mesures avec une énergie incidente aussi faible que possible et appliquée pendant une durée aussi faible que possible. Ceci nécessite pour le galvanomètre de mesure une grande sensibilité et une courte période, deux qualités contradictoires. Heureusement la difficulté peut être tournée par l'emploi d'un amplificateur galvanométrique à contre-réaction. Le galvanomètre primaire pourra être à sensibilité élevée, grande résistance interne et grande période, dans la mesure où ces caractéristiques ne nuiront pas à sa stabilité. L'emploi d'un taux de contre-réaction convenable permettra un accroissement notable de sensibilité tout en réduisant considérablement l'impédance d'entrée et la constante de temps. Dans ces conditions il est possible d'obtenir des mesures parfaitement reproductibles. P'our chaque température on pourra donc relever une courbe de sensibilité spectrale bien définie, représentée par la relation : $\sigma_{T \lambda}=l(\lambda)$. Le réseau de courbes obtenues est représenté par l'ensemble de courbes de la ligure 1. 
B. Paramètres propres au rayonnemen't de RESENSIBILISATION : $\lambda_{R}-\theta_{R}-W_{R \lambda}$ - La sensibilité obtenue au cours de réactivation $\sigma_{\lambda}$ dépend de la longueur d'onde de réactivation, de son inten-

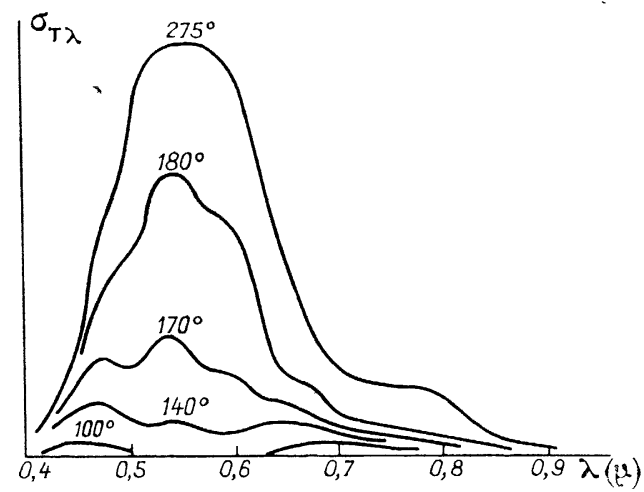

Fig. 1. - Courbes de sensibilité spectrale vraie.

sité et de la durée de réactivation. Toutefois la sensibilité limite que l'on peut obtenir pour une longueur d'onde $\lambda$ ne dépend que de la température de refroidissement : c'est la phase stable dont nous avons parlé précédemment. En cherchạnt pour chaque mesure à obtenir cette sensibilité limite il est possible de tracer pour chaque température $T$ une courbe de sensibilité spectrale reproductible, $\sigma_{\infty \lambda}=g(\lambda)$.

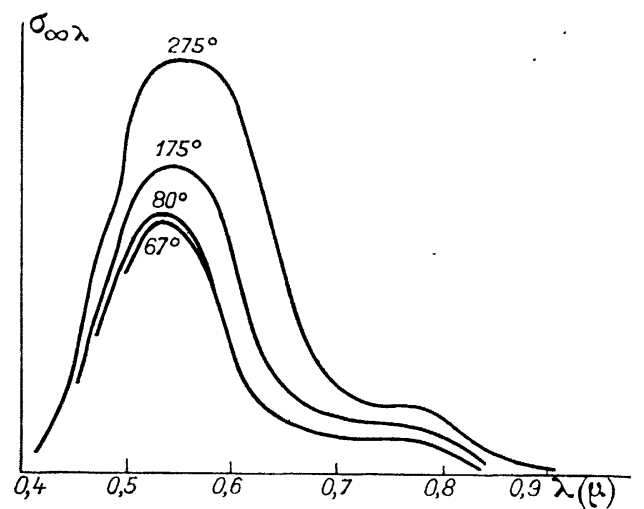

Fig. 2. - Courbes de sensibilité spectrale après réactivation totale.

Le réseau des courbes obtenues est tracé sur la figure 2.

Ces résultats peuvent également être représentés de la manière suivante : en portant en ordonnées le rapport de la sensibilité $\sigma_{\infty \lambda}$ à To à la sensibilité $\sigma_{0 \lambda}$ à $273^{\circ} \mathrm{K}$ pour la même longueur d'onde. On obtient ainsi les courbes des figures 3 et 3 bis relatives respectivement à deux types de cellule.

Pour une longueur d'onde donnée, on peut tracer les courbes de variation de $\sigma_{T \lambda}$ et de $\sigma_{\infty \lambda}$ en fonction de la température. Étant donnée la différence considérable d'amplitude des variations de ces deux sensibilités, nous avons représenté en unité arbi- traires les variations de $\sigma_{T \lambda}$ avec une échelle logarithmique et les variations de $\sigma_{\infty \lambda}$ avec une échelle linéaire. La figure 4 est relative à la longueur d'onde $0,5 \mu$.

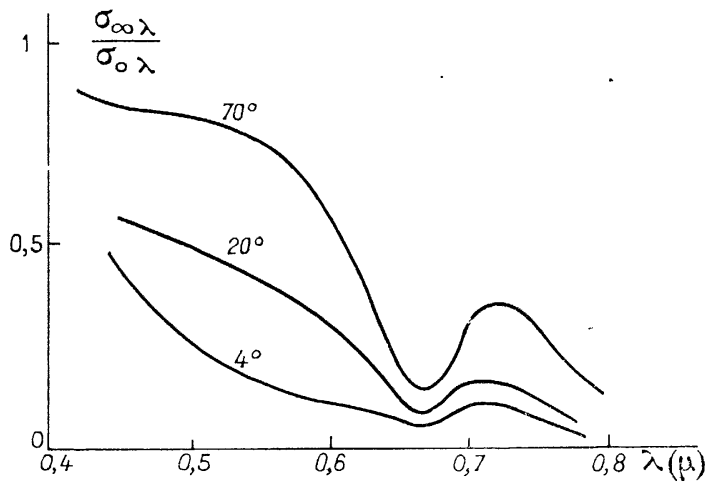

FIG. 3. - Baisse de sensibilité par rapport à $273^{\circ} \mathrm{K}$ pour deux types de cellule après réactivation.

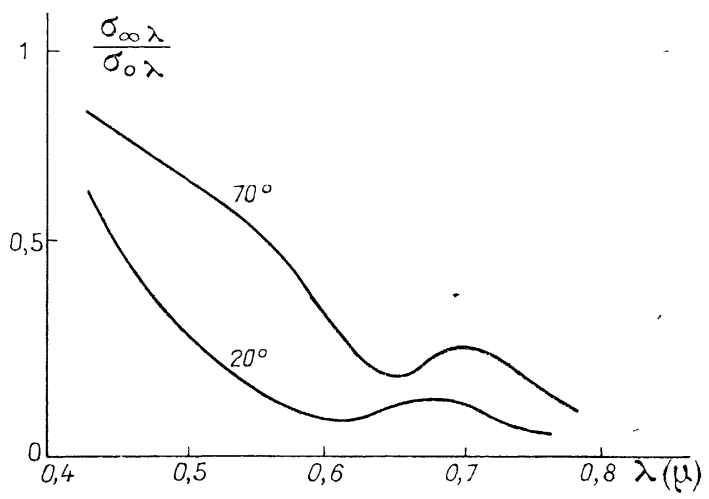

Fig. 3 bis. - Baisse de sensibilité par rapport à $273 \circ \mathrm{K}$ pour deux types de cellule après réactivation.

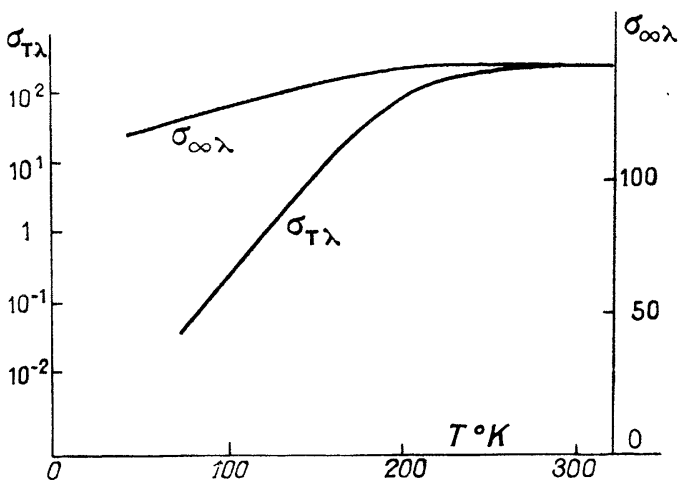

Fig. 4. - Variation en fonction de $T$ de la sensibilité vraie et après réactivation totale pour $\lambda=0,5 \mu$.

Pour $T=273 \circ \mathrm{K}$, le phénomène de resensibilisation n'existant pas, les deux sensibilités ont la même valeur. Le tableau ci-dessous donne les valeurs numériques à la figure 4.

$\begin{array}{crrrrrrrrr}T & 273 & 250 & 225 & 200 & 175 & 150 & 125 & 100 & 75 \\ \sigma_{T \lambda} & 139 & 137 & 135 & 105 & 32 & 3 & 1 & 0,25 & 0,05 \\ \sigma_{\infty \lambda} & 139 & 139 & 139 & 137 & 134 & 128 & 121 & 116 & 110\end{array}$


La décroissance de la sensibilité $\sigma_{q^{\prime} \lambda}$ peut être représentée approximativement (pour les températures inférieures à $175 \mathrm{oK}$ ) par une relation du type :

$$
L \sigma_{T \lambda}=A+B T .
$$

a. Influences respectives de l'intensité et de la durée du rayonnement de réactivation: $W_{R \lambda}$ et $\theta_{R}$. Ces influences étant les mêmes qualitativement quels que soient $\lambda, \lambda_{R}$ et $T$, nous exposerons les résultats numériques obtenus avec $\lambda=0,55 \mu-\lambda_{R}=0,75 \mu$ et $T=100^{\circ} \mathrm{K}$. Pour diverses valeurs de l'intensité, nous avons fait croître progressivement la durée et relevé simultanément l'évolution de la sensibilité. Nous avons constaté que les effets sont cumulatifs et que la sensibilité obtenue $\sigma_{\lambda}$ ne dépend que du produit : $W_{R \lambda} \cdot \theta_{R}=E_{R \lambda}$.

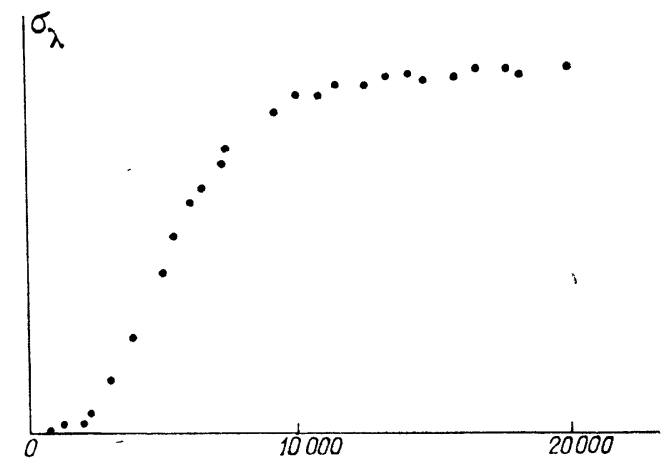

Fig. 5. - Récupération de la sensibilité en fonction de l'énergie $I R$ reçue.

Points expérimentaux.

La figure 5 montre comment se situent les points représentatifs de $\sigma_{\lambda}$ pour diverses valeurs de $E_{R \lambda}$ obtenues par combinaison des valeurs de $W_{R \lambda}$ et de $\theta_{R}$.

$W_{R \lambda}$ a varié de 0,18 à 1,34 microwatts.

$\left\{\theta_{R} \quad\right.$ a varié de 1 à 25 minutes.

$E_{R \lambda}$ a donc varié de 100 à 20000 ergs.

Aux erreurs d'expérience près, ces points se situent sur une courbe unique, ce qui prouve que $W_{R \lambda}$ et $\theta_{R}$ n'interviennent que par leur produit; autrement dit, seule compte pour la resensibilisation l'énergie globale reçue par la cellule. Cette loi de sommation est encore valable pour des éclairs $I R$ de durée $1 / 100$ e de seconde.

La courbe de la figure 5 peut donc se déterminer avec un rayonnement d'intensité connue dont on fait varier la durée d'application.

Ainsi avec $\lambda_{R}=0,90 \mu$ et des durées croissantes de 3 en 3 secondes, nous avons déterminé la courbe de la figure 6 avec une centaine de points. Cette courbe met en évidence au début une phase de latence, puis une phase à croissance rapide et enfin une phase de saturation. L'intensité du rayonnement était de 2 microwatts. b. Influence de la longueur d'onde du rayonnement de réactivation $\lambda_{R}$. - Nous avons constaté que pour une même intensité du rayonnement $\lambda_{R}$, la vitesse de réactivation dépendait de cette longueur d'onde.

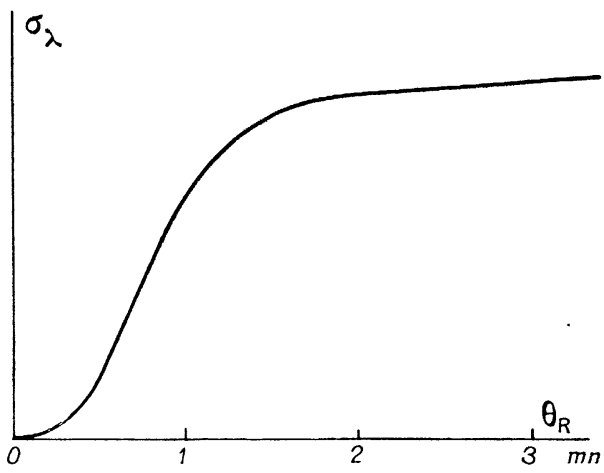

FIG. 6. - Récupération de la sensibilité en fonction de l'énergie $I R$ reçue.

Courbe expérimentale moyenne tracée avec plus de cent points.

$$
\begin{aligned}
W_{R} & =2 \mu \mathrm{W} \\
\lambda_{R} & =0,9 \mu .
\end{aligned}
$$

Si l'on trace les courbes analogues à celle de la figure 6 , pour diverses longueurs d'onde, elles sont très différentes les unes des autres. Toutefois et ceci est très intéressant, ces diverses courbes peuvent être superposées presque rigoureusement si l'on choisit pour chacune d'elles une unité de temps convenable sur l'axe des abscisses. La longueur figurant l'unité de temps sur l'axe des abscisses sera donc d'autant plus longue que la radiation considérée sera plus efficace pour réactiver la cellule. Elle peut donc servir à mesurer cette efficacité. La détermination de la pseudo constante de temps de ces diverses courbes conduit au même résultat.

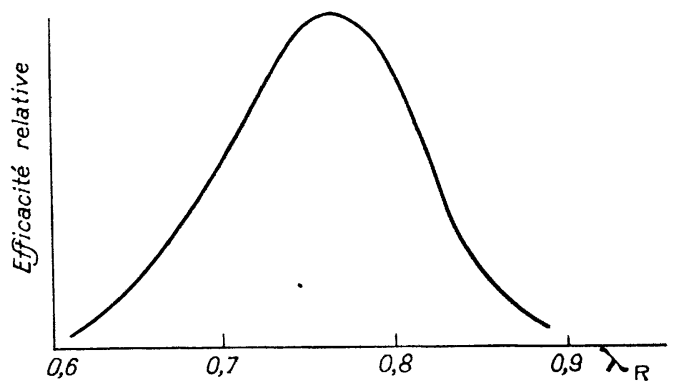

FIG. 7. - Distribution spectrale

de l'efficacité de réactivation en valeur relative.

Les résultats sont rassemblés dans le tableau suivant :

$\begin{array}{llllllll}\lambda_{R} & 0,60 & 0,65 & 0,70 & 0,75 & 0,80 & 0,85 & 0,90 \\ \text { relative.... } & \frac{1}{10} & \frac{0}{16} & \frac{24}{34} & \frac{1}{8} & \frac{1}{1}\end{array}$

$\begin{array}{llllllll}\text { Efficacité relative.... } & 1 & 10 & 16 & 24 & 34 & 8 & 1\end{array}$

Ils peuvent être représentés par la courbe de la figure 7. 
¿. Influence de la temperature : $T_{n} \ldots$ Si la température de rofroidissement $T_{R}$ est plus élevée, Ja sensibilité $\sigma_{\lambda}$ atteint plus rapidement sa valeur limite que si $T_{R}$ est plus bas, pour des caractéristiques identiques du rayonnement de réactivation. Cette fois encore une seule courbe permet de représenter les résultats aux diverses températures en portant en ordonnée $\sigma_{\infty} \sigma_{\infty \lambda}$ et en abscisse

l'énergie de réactivation aver mone échelle dépendant de la température.

III. Résultats d'ensemble. Le trait raractéristique de ces pluénomines de réactivation est qu'une courbe unique permel de le représenter dans

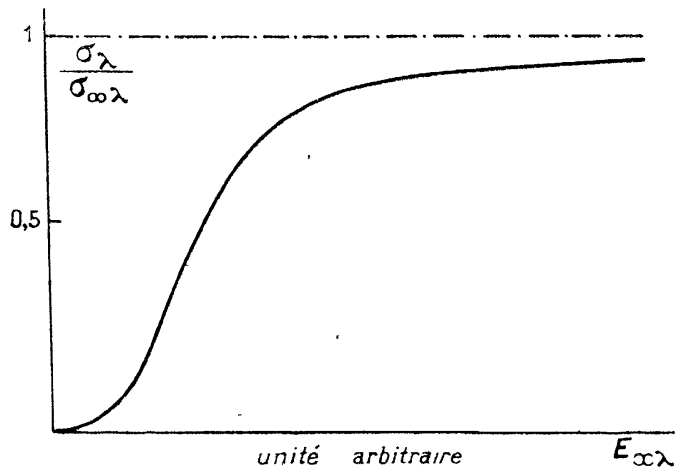

Fic. 8. - Récupération de la sensibilité en fonction de l'énergie $I R$ reçue.

Sensibilité en valeur relative ; énergie en unité arbitraire.

loutes ses particularités. Ciette courbe a été tracée avec précision sur la figure 8 . Cette courbe est celle de la figure 6 , mais nous avons choisi une unité arbitraire sur l'axe des abscisses. En abscisses sont portées les énergies de réactivation et en ordonnées le rapport de la sensibilité à la sensibilité réactivée limite.

Cette fonction standard est de la forme:

$$
\sigma_{\lambda}=F\left(E_{R \lambda}\right) \cdot \sigma_{\infty} \lambda \text {. }
$$

linfluence de la longueur d'onde $\lambda$ se traduit par la présence d'un coefficient $\Lambda_{1}(\lambda)$ en facteur au second membre puisque la sensibilité finale $\sigma_{\infty}$. dépend de $\lambda$ (voir fig. 2) ; bien que la vitesse de réactivation soit indépendante de $\lambda$. L'influence de la température se manifeste par la présence de deux coefficients : l'un $B_{1}(T)$ en facteur au second inembre puisque $\sigma_{\infty \lambda}$ dépend de $T$ (voir fig. 2), et l'autre $B_{2}(T)$ en facteur de $E_{R}$ car la vitesse de réactivation dépend de la température.

En effet, si nous cherchons la valeur de la durée nécessaire pour réactiver à la limite $\left(\sigma_{\infty \lambda \lambda}\right)$ à intensité $\left(W_{R}\right)$ constante. nous trouvons des durées d'autant plus grandes que $T$ est plus faible. et dans la zone de 75 à $175^{\circ} \mathrm{K}$. la variation est pratiquement exponentielle ainsi que le montre la figure 9

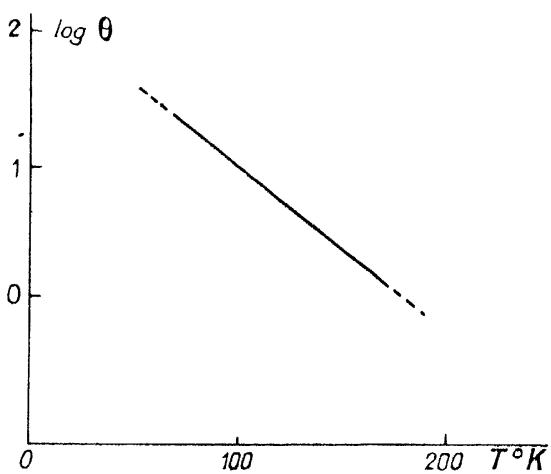

Fir. 9. Variation, en fonclion de $T$, de la durée nécessaire pour une réactivation lolale avec $\lambda_{B}=0,75 \mu$.

La durée nécessaire arec 2 microwatts de $\lambda_{R}=0,75 \mu$ passe de 5 à 180 secondes lorsque $T$ diminue de 175 à $75^{\circ} \mathrm{K}$, pour $\lambda=0,50 \mu$.

L'influence enfin de la longueur d'onde de réactivation $\lambda_{l l}$ se traduisant par une variation de la vitesse de réactivation se traduira par un coefficient $C^{\prime}\left(\lambda_{R}\right)$ en facteur de $E_{R}$.

L'expression générale est done:

$$
\sigma_{\lambda}=l_{1}(\lambda) B_{1}(T) F^{\prime}\left[B_{2}(T) C\left(\lambda_{R}\right) E_{L \lambda \lambda} \mid\right. \text {. }
$$

IV. Influence du courant de mesure. ... I)ans tout ce qui précède nous avons déterminé la sensibilité de la cellule à courant constant. En effet, il est commode de conserver un même appareil de mesure pour toutes les séries de mesure et d'agir sur les réglages du monochromateur pour avoir des déviations comparables quel que soit $\lambda$. Ceci ne va pas sans inconvénient car nous représentons les variations de sensibilité par des courbes supposées implicitement à énergie constante : or si la cellule n'a pas une caractéristique linéaire, il n'y a pas correspondance entre la courbe tracée et les résultats de mesure.

Bien que les précautions classiques aient été prises : éclairement faible, résistance de charge faible, etc..., il apparaît aux basses températures, pour une cellule non réactivée ou en cours de. réactivation, des écarts considérables à la linéarité. Ces écarts se traduisent par le fait que la courbe représentant la fonction standard:

$$
\sigma_{\lambda}-F\left(E_{\lambda \lambda .}\right) \cdot \sigma_{\infty}
$$

dépend du courant débité par la cellule, donc de l'éclairement qui sert à la détermination de la sensibilité.

Tout ce que nous avons dit de la permanence et de l'universalité de cette fonction subsiste tant que le courant de mesure / est le même. Pour une autr" valenr $l^{i}$ de ce rourant, rette fonction prend une 
valeur différente $F^{\prime \prime}$ qui reste miversello lant que $l$ ' yarde sa valeur.

Nous avons déterminé la forme de la fonction $l i$ pour quatre courants différents ayant pour valeurs respectives en microampères :

$$
I_{1}=0,0006 ; I_{2}=0,006 ; I_{3}=0,06 ; I_{4}=0,6 .
$$

('ette valeur de courant correspond au courant" maximum obtenu après réactivation complìte pour la longueur d'onde de 0,50 y à la température de 100 ok. (Réactivation aver is $=0,75$, $W_{P \lambda}=0,2 \mu H^{\top}$.

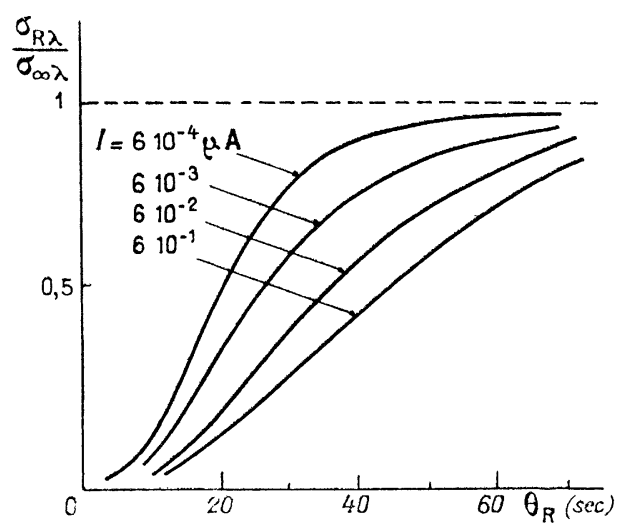

Fig. 10. - Courbes de réactivation pour quatre valeurs différentes du courant de mesure.

$$
\begin{aligned}
\lambda_{R} & =0,75 \mu \\
W_{n \lambda} & =0,2 \mu W \\
T & =100 \% \mathrm{~K} .
\end{aligned}
$$

Jes quatre courbes obtenues sont reproduites sur la figure 10 .

I'n fait important (que l'on ne voit pas sur la figure puisque les coordonnées sont en valeur relative) est que la limite $\sigma_{\infty \lambda}$ ne dépend pas du courant de mesure et représente toujours la même fraction de la sensibilité $\sigma_{0 \lambda}$.

Ces courbes présentent toujours le mème aspect à trois phases : phase de latence. phase de croissance linéaire ot phase de saturation.

Le fait remarquable est encore que ces diverses fonctions $F, F^{\prime}$, etc... sont divers aspects d'une même fonction universelle ainsi que le montre la figure 11 sur laquelle les points expérimentaux de la figure 10 ont été transposés en choisissant une échelle des temps différente pour chaque rourbe.

Si l'on cherche la durée de réactivation - pour laquelle la cellule est réactivée à un pourcentage donné de la valeur limite, on constate que cette durée est liée à l'intensité $I$ du courant de mesure (l pour la sensibilité limite) par une relation très simple du type :

$$
=-1 \mid B I,(I) \text {. }
$$

Cette formule est au moins valable dans les limites de notre expérimentation. c'est-à-dire pour des courants rariant de 1 i 1000 entere les limites préciséescion-dessits.

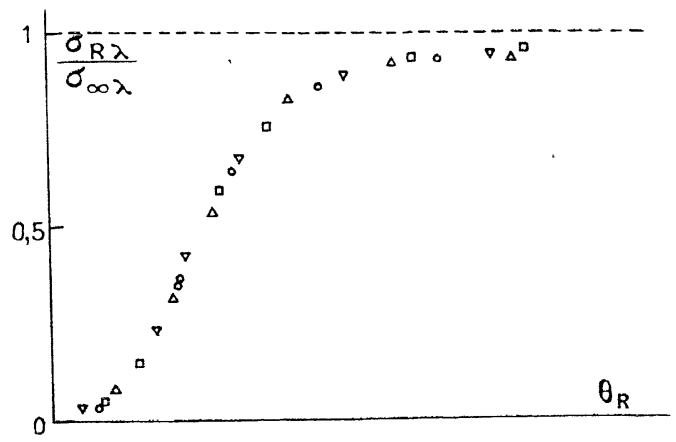

Fra. 11.... Réduction à une courbe uniquue des courbes de la figure 10.

$$
\begin{aligned}
& 1-610^{-1} \text { u.l } \\
& \text { (6) } 10^{-3} \\
& \text { f } 10^{-} \cdot \\
& \text { (i) } 10.1 \text { II }
\end{aligned}
$$

Fin lail $\tau$, ou l'énergie de réactivation nécessaire. croit donc heaucoup moins vite que le courant de mesure. Lorsque celui-ci est multiplié par 1000 . - est seulement multiplié par 2,30 , dans le domaine considéré. Il est bien évident toutefois que si la formule ci-dessus représente correctement l'expérience dans un certain domaine, elle ne peut convenir ni pour les courants de mesure très faibles, ni pour les courants de mesure très grands.

\section{$V$. Influence du mode de refroidissement.} Nous avons essayé d'amener la cellule à la température $T$ de plusieurs manières différentes :

a) Refroidissement direct le plus rapide possible, soit de quelques minutes.

b) Refroidissement direct Ient. soit une heure environ.

(c) Refroidissement diroct à une température $T^{\prime} \leqslant T$, maintenu pendant un temps plus ou moins long à $T^{\prime}$. puis réchauffoment plus ou moins rapide ¿े $T$.

d) Gucle thermicfue Jautaisiste amenant finalement la cellule à la température T'.

Dans tous les cas la courhe de réactivation est la même et caractérise done la température $T$, indópendamment du excle thermique parcouru auparavant par la celluile.

VI. Mesures aux très basses températures. II nous a été possible de reprendre une partie de ces mesures aux températures d'ébullition, sous la pression atmosphérique de l'hydrogène et de I'hélium liquide, soit respectivement $20^{\circ}$ et $/ \mathbf{t}{ }^{\circ} \mathrm{K}$. Toutefois notre tube laboratoire n'était vraisemblablement pas en équilibre thermique et ces températures ne sont que des ordres de grandeur. Les phénomènes de réactivation se produisent à ces 
températures comme aux autres et les résultats expérimentaux sont tout ì lait comparables ainsi qu'il ressort de l'examen des quelques courbes ci-après qui ont été déterminées pour deux types de cellule, $\mathrm{A}$ et $\mathrm{B}$, de provenance différente.
VII. Stabilité d'une cellule réactivée. - La réactivation partielle ou totale d'me cellule est un phénomène stable, ou plus exactement métastable. Nous avions signalé par ailleurs une lente décroissance de la sensibilité après réactivation. Cette a. Sensibilité spectrale.
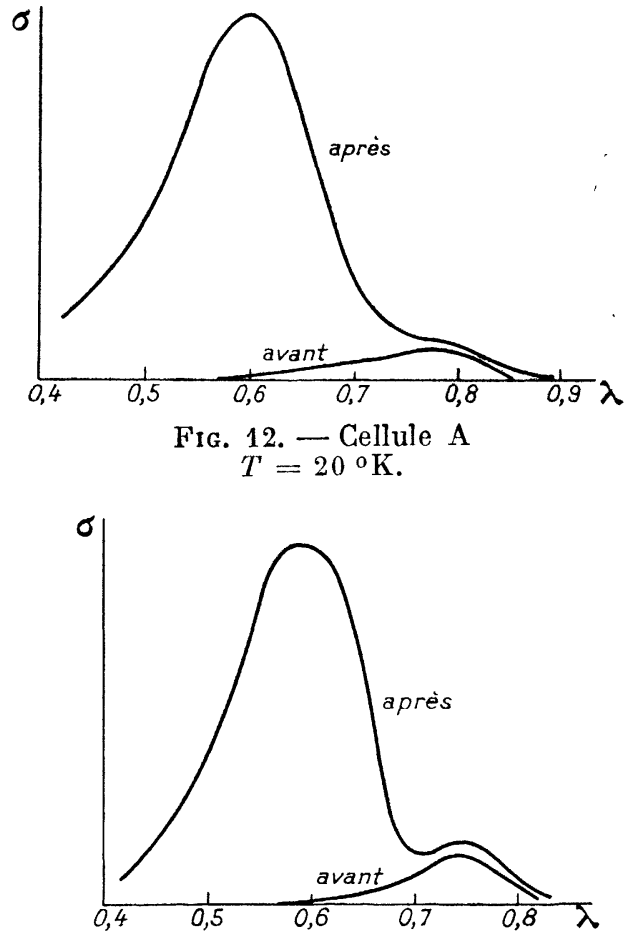

Fig. 13. - Cellule B $T=20^{\circ} \mathrm{K}$.

\section{b. Réactivation.}

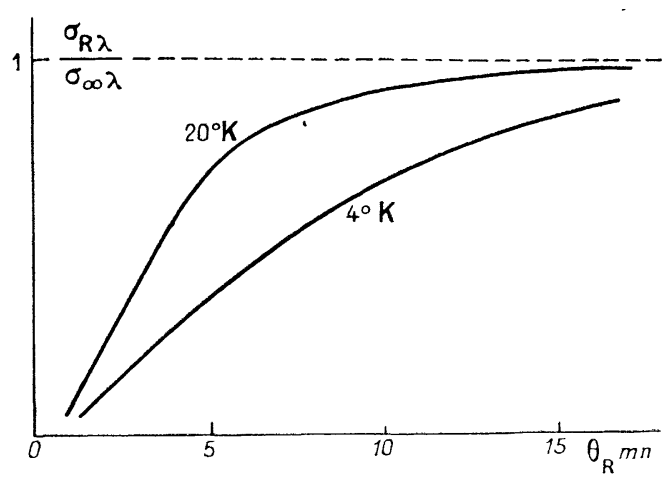

Fig. 14. - Cellule A.

Courbes ramenées à la même asymptote.

particularité était sans doute à imputer à un abaissement de température de la cellule par suite d'un déséquilibre thermique entre cette dernière et le cryostat. En prenant la précaution d'opérer à une température parfaitement définie, la décroissance

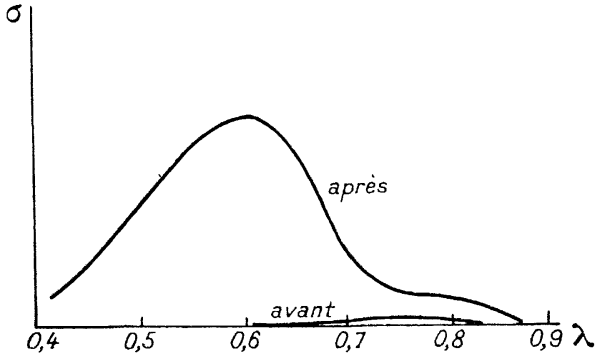

FIg. 12 bis. - Cellule $\Lambda$ $T=4^{\circ} \mathrm{K}$.

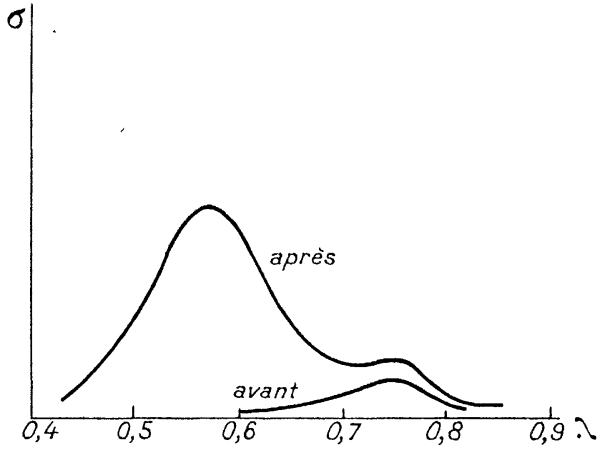

Fig. 13 bis. - Cellule B $T=4 \circ \mathrm{K}$.

\section{c. Répartition spectrale}

de l'activité resensibilisatrice.

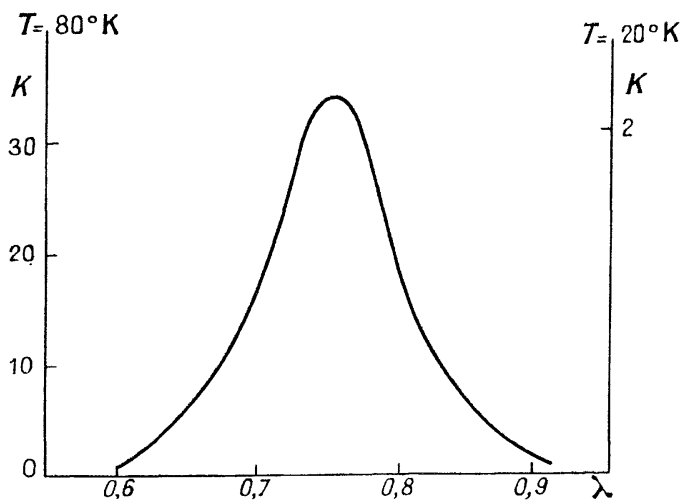

Fig. 15. - $\boldsymbol{K}$, facteur par lequel la sensibilité est multipliée pour $\lambda_{0}=0,55$ après 10 secondes d'éclairage par $\lambda$. Remarquer les différences d'échelles de $K$ pour $T=80^{\circ} \mathrm{K}$ $\grave{a}$ gauche et $T=20 \mathrm{oK}$ à droite. Les courbes sont confondues.

de sensibilité, si elle existe, est limitée à quelques pour cent en plusieurs heures. Par contre, on peut faire subir à une cellule du cycle un type suivant 
(la sensibiliti esl mesurée en valeurs arbitraires pour la longueur d'onde $\lambda_{0}=0,55 \mu$ ):

a) Cellule à $273^{\circ} \mathrm{K} \ldots \ldots \ldots \ldots \ldots, \sigma=140$

b) Cellule à $750 \mathrm{~K} \ldots \ldots \ldots \ldots \ldots \ldots \ldots, \quad \sigma<0,1$

c) Réactivation à $75^{\circ} \mathrm{K}$ par 80 secondes d'éclairement $\lambda=0,75 \mu$ $W=0,2 \mu \mathrm{W} \ldots \ldots \quad \sigma=48$

d) Cellule à $64^{\circ} \mathrm{K}$ pendant une heure . $\sigma=13$

e) Cellule à $100^{\circ} \mathrm{K}$ pendant 10 minutes. $\sigma=110$

f) Cellule à $75 \circ \mathrm{K} \ldots \ldots \ldots \ldots \ldots, \sigma=47$

$\mathrm{Au}$ petit écart près entre 47 et 48 (et peut-être dû à une petite différence de température), ce cycle thermique n'a pas modifié la sensibilité de la cellule partiellement réactivée à 75 oK. Mais cet état n'est que métastable car il suffit d'une nouvelle réactivation, ou d'un réchauffement au-dessus de $175^{\circ} \mathrm{K}$, pour le modifier d'une façon irréversible.

Conclusions. - Nous avons mis en évidence un phénomène nouveau, la resensibilisation par rayonnement du proche infrarouge d'une photopile au sélénium préalablement refroidie. Nous avons étudié isolément chacun des nombreux paramètres qui conditionnent ce phénomène et avons abouti aux résultats suivants :

a) Le domaine d'efficacité des radiations resensi- bilisatrices s'étend de $0,6 \mu$ à $0,9 \mu$, avec un maximum vers $0,77 \mu$.

b) La courbe de réactivation (sensibilité, temps) peut se ramener à une courbe type unique en choisissant sur les deux axes des unités dépendant des paramètres de façon convenable ; nous avons déterminé expérimentalement ces lois.

c) Une cellule non activée ou partiellement réactivée est dans un état métastable pouvant s'orienter vers un état semi-stable, cellule totalement réactivée, par envoi d'une dose suffisante d'infrarouge, ou vers un état stable, l'état normal, par réchauffement à la température ambiante.

L'explication théorique de cet ensemble de phénomènes est en cours d'étude. Elle repose essentiellement sur le mécanisme de piégeage que nous avons exposé par ailleurs, sur l'action de la température sur les probabilités de capture et sur le dépeuplement de certains niveaux d'énergie sous l'action d'un refroidissement.

Les mesures dans le domaine de 20 o K et 4 o K ont été réalisées à l'Institut Fourier à Grenoble, grâce à l'obligeance de M. Néel que je remercie tout particulièrement.

Manuscrit reçu le 22 octobre 1956.

\section{BIBLIOGRAPHIE}

[1] Buet (G.), L'effet photoélectrique des photopiles au sélénium aux basses températures. C. R. Acad. Sc., 1955, 240, 962-963.

[2] Blet (G.), Modification de la courbe de sensibilité spectrale des photopiles au sélénium sous l'action des variations de température. C. R. Acad. Sc., 1956, 242, 95-98.

[3] BLeT (G.), De l'influence du proche infrarouge aux basses températures sur les propriétés des photopiles au sélénium. C. R. Acad. Sc., 1956, 243, 798-800. 